\title{
Search for a neutrino flux from the Fermi Bubbles with the ANTARES telescope
}

\author{
S. Hallmann* on behalf of the ANTARES collaboration. ${ }^{\dagger}$ \\ University of Erlangen-Nürnberg, Erwin-Rommel-Str. 1, 91058 Erlangen, Germany \\ Erlangen Centre for Astroparticle Physics (ECAP) \\ E-mail: steffen.hallmannefau.de
}

The Fermi Bubbles are two giant lobes of $\gamma$-ray emission above and below the Galactic Center. Whereas the origin of the observed $\gamma$-ray flux remains obscure, the measurement of a neutrino flux from the Fermi Bubbles could distinguish between leptonic and hadronic emission scenarios. Such a search for a neutrino signal from the Fermi Bubbles has been performed with the ANTARES neutrino telescope in the Mediterranean Sea using four years of data. The search has used charged current muon neutrino interactions, which produce muons with long tracks in the detector and therefore have an angular resolution of well below one degree. In the analysis, the background is determined from off-regions and compared to the number of events observed in the Fermi Bubble zone. The results of an update using data from 2012 and 2013 are presented. Since no statistically significant excess was found the new upper limits for six years of ANTARES data are presented.

The 34th International Cosmic Ray Conference,

30 July - 6 August, 2015

The Hague, The Netherlands

\footnotetext{
*Speaker.

${ }^{\dagger}$ http://antares.in2p3.fr
} 


\section{Introduction}

The Fermi-LAT experiment has revealed two giant lobes of $\gamma$-ray emission extending $7-8 \mathrm{kpc}$ $\left(\approx 50^{\circ}\right)$ above and below the Galactic Centre [1]. These are commonly referred to as the Fermi Bubbles (FB). Structures in spatial correlation with the FB have also been observed in X-rays [2], in the microwave band [3] and radio-wave band [4]. To date the origin of the FB remains unknown. Several proposed models explaining the emission include hadronic mechanisms, in which the $\gamma$ rays together with a corresponding neutrino signal are produced by the collisions of cosmic-ray protons with interstellar matter $[5,6,7]$. In contrast, models based on leptonic mechanisms or dark matter decay would yield less neutrino emission or none at all $[1,6,8,9]$. The observation of a neutrino signal from the FB region would therefore give a unique possibility to discriminate between the different models.

A search for a signal from the Fermi Bubbles with the ANTARES neutrino detector with four years of data (2008-2011) has already set an upper limit on the neutrino flux [10]. The analysis used off-zones with same visibility in the ANTARES detector to determine the background in the Fermi Bubbles' region. In the signal region a statistically insignificant excess of $1.2 \sigma$ over the background was observed. In this proceeding, the result of an update on the existing analysis using two additional years of data taking (2012 \& 2013) is presented.

The ANTARES telescope $[11,12]$ is a deep-sea neutrino detector located $40 \mathrm{~km}$ off Toulon (France) taking data in its final configuration since 2008. In the search for a neutrino signal from the FB muons and neutrinos emerging from cosmic-ray interactions in the atmosphere constitute the main backgrounds. While the water overburden acts as a partial shield, the rate of atmospheric muons coming from above the detector still dominates over the neutrino signal. Signal searches reduce this background by looking only at events coming from below the detector. The cosmic signal is distinguished from atmospheric neutrinos by its harder energy spectrum. A cut on the reconstructed energy exploits this feature.

This analysis focusses on charged current interactions of muon neutrinos $\left(v_{\mu}+\bar{v}_{\mu}\right)$. In this interaction channel a relativistic muon is produced and emits Cherenkov light along its path through the water. The direction is reconstructed by maximising a likelihood which fits the photon arrival times at the optical modules (hits) to the Cherenkov emission on the hypothesised muon track. This gives a median angular resolution on the neutrino direction of $0.46^{\circ}$ [13].

Thanks to the detector position at $43^{\circ}$ latitude in the northern hemisphere, ANTARES has an excellent visibility to the region around the Galactic Centre. Their position hence makes the Fermi Bubbles an ideal target to look for galactic neutrino emission.

\section{Spectrum of the expected neutrino flux from the Fermi Bubbles}

Fig. 1 shows the shape of the $\gamma$-ray lobes observed with Fermi-LAT. They show a relatively uniform $\gamma$-ray emission over the whole region [1]. Ref. [1] measured a hard $\gamma$-ray spectrum without visible cutoff compatible with a power-law $E^{-\alpha}$ with spectral index $\alpha=2$, and a corresponding flux of

$$
E^{2} \frac{\mathrm{d} \Phi_{\gamma}}{\mathrm{d} E} \approx 3-6 \times 10^{-7} \mathrm{GeV} \mathrm{cm}^{-2} \mathrm{~s}^{-1} \mathrm{sr}^{-1}
$$




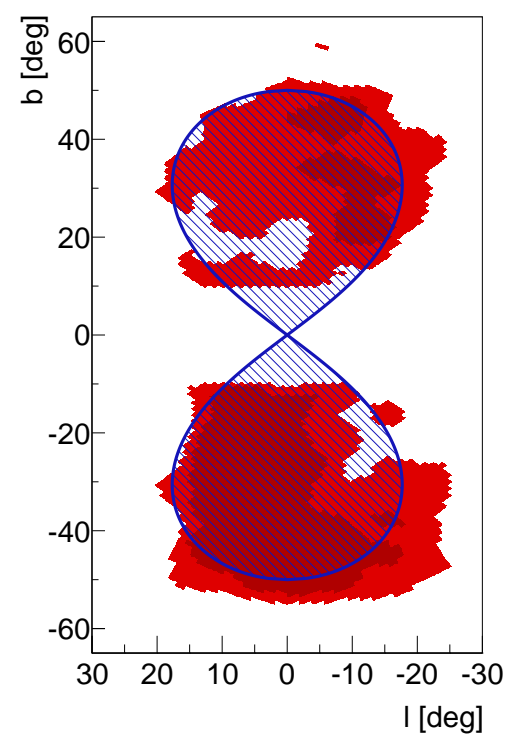

Figure 1: The geometric shape used in the analysis (shown in blue) has a good overlap with the shape of the FB structure found in Ref. [14] (indicated in red). Especially the 'cocoon' structure (dark red), which shows a higher $\gamma$ intensity, is well covered (91\%).

A more recent study by Fermi-LAT on the spectrum of the $\gamma$-rays prefers steeper spectra or low cutoff energies. Using the $\gamma$-flux parametrisation from the SYBILL-code from Ref. [15], it is shown in Ref. [16] that an $E^{-2.25}$ proton spectrum can produce a $\gamma$-flux that fits the Fermi-LAT data well. A power-law fit to this parametrisation at energies beyond $10 \mathrm{GeV}$ yields a spectral index of $\alpha=2.18$ and a $\gamma$-flux (c.f. [16, Fig. 2])

$$
E^{2.18} \frac{\mathrm{d} \Phi_{\gamma}}{\mathrm{d} E} \approx 0.5-1.0 \times 10^{-6} \mathrm{GeV}^{1.18} \mathrm{~cm}^{-2} \mathrm{~s}^{-1} \mathrm{sr}^{-1} .
$$

In a purely hadronic emission scenario a $\gamma$-ray flux and a corresponding neutrino flux are generated by the decay of neutral and charged pions, which emerge from the interaction of cosmicray protons with the interstellar gas [15]. At high energies the neutrino and $\gamma$-ray flux in this hadronic case differ only by a scaling factor $\xi\left(\alpha_{\gamma}\right)$ [17],

$$
\Phi_{v}(E)=\xi\left(\alpha_{\gamma}\right) \times \Phi_{\gamma}(E) .
$$

The scaling depends on the spectral index of the $\gamma$-rays, $\alpha_{\gamma}$, and is $\xi\left(\alpha_{\gamma}\right) \approx 0.41(0.36)$ for an $E_{\gamma}^{-2}$ $\left(E_{\gamma}^{-2.18}\right)$ spectrum [17].

The Fermi satellite due to its limited size can only measure the photon spectrum to energies of some $100 \mathrm{GeV}$. The spectrum and cutoff of the FB signal at higher energies is to date undetermined. Within our galaxy it is however assumed that protons can only be efficiently accelerated up to energies of 1-10 PeV [5]. This will induce also a cutoff in the observed $\gamma$-ray and neutrino spectra. As a crude approximation $20 \%$ of the proton energy is on average converted into charged pions. An equal distribution over the four daughters in pion decay yields

$$
E_{v}^{\text {cutoff }}=0.05 \times E_{p}^{\text {cutoff }}
$$

for the neutrino cutoff, i.e. cutoffs in the range of $50-500 \mathrm{TeV}$. Combining Eq. 2.1 with an exponential cutoff from Eq. 2.4 and taking into account the scaling factor yields the expected 
Figure 2: Hammer equal-area map projection in galactic coordinates showing the on-zone and off-zones. The shaded area is the Fermi Bubbles region (on-zone). The three off-zones are shifted by 6,12 and 18 hours in time. The colour scale represents the visibility of the sky at the ANTARES site ranging from $0 \mathrm{~h}$ (white) to $24 \mathrm{~h}$ (blue) per day. Fig-

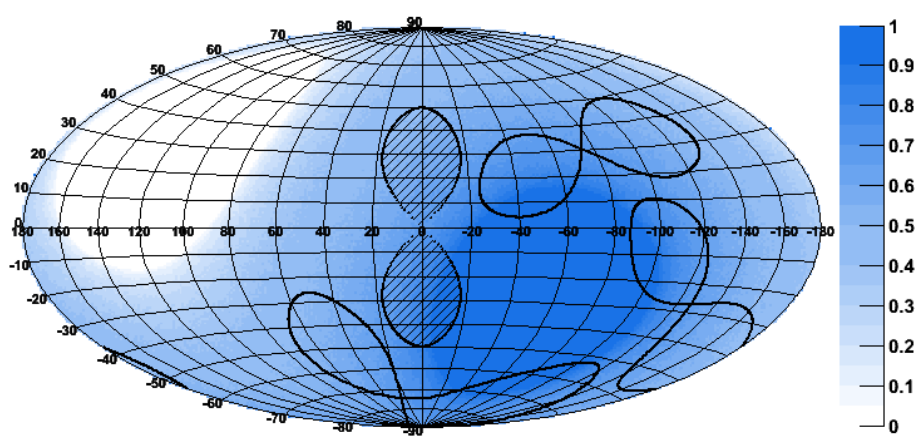
ure taken from Ref. [20].

neutrino fluxes,

$$
\begin{gathered}
E^{\alpha} \frac{\mathrm{d} \Phi_{v_{\mu}+\bar{v}_{\mu}}}{\mathrm{d} E}=A_{\text {model }}^{\alpha} \times \exp \left(-\frac{E}{E_{V}^{\text {cutoff }}}\right), \\
A_{\text {model }}^{2.0}=1.2-2.4 \times 10^{-7} \mathrm{GeV} \mathrm{cm}^{-2} \mathrm{~s}^{-1} \mathrm{sr}^{-1} \quad \text { for } \alpha=2.0,
\end{gathered}
$$

and similarly for the flux assumption from Eq. 2.2,

$$
A_{\text {model }}^{2.18}=1.8-3.6 \times 10^{-7} \mathrm{GeV}^{1.18} \mathrm{~cm}^{-2} \mathrm{~s}^{-1} \mathrm{sr}^{-1} \quad \text { for } \alpha=2.18
$$

\section{Event selection for the on-/off-zone analysis}

For the analysis a preliminary event selection is applied on the data to reject badly reconstructed events and background: To reject most of the atmospheric muons only events reconstructed as up-going are selected. Events are kept, if the track fit algorithm used more than 10 hits. A cut on a parameter describing the angular error of the reconstruction, $\beta<1^{\circ}$, deselects events with misreconstructed directions. Shower-like events are identified by an alternative $\chi^{2}$-based fit algorithm. This algorithm assumes the hypothesis of a showering event signature $\left(\chi_{\text {point }}^{2}\right)$ and that of a muon track $\left(\chi_{\text {track }}^{2}\right)$. Events which are shower-like $\left(\chi_{\text {point }}^{2}<\chi_{\text {track }}^{2}\right)$ are excluded from the analysis.

The optimisation of the event selection is done on two parameters: The track fit quality $\Lambda$, and the reconstructed energy $E_{\text {reco. }}$. The cut on $\Lambda$ is mainly used to reject atmospheric muons. The energy estimate $E_{\text {reco }}$ is determined by Artificial Neutral Networks. To produce these a machine learning algorithm was trained to derive an energy estimate [18] from a set of variables, such as the number of detected photons and the total charge deposited on the optical modules. For $10 \mathrm{TeV}$ muons the median resolution is $30 \%$ on $\log _{10}\left(E_{\text {reco }}[\mathrm{GeV}]\right)$.

A blind strategy is adopted for the analysis in which the optimisation of the cuts on $\Lambda$ and $E_{\text {reco }}$ is performed using simulated signal and background data only.

For the signal search the number of events originating from the combined region of the two FB lobes above and below the Galactic Centre (on-zone) is compared to the background observed in regions from which no signal is expected (off-zones). A simplified shape of the FB, which approximates the template area identified in Ref. [14], is used for the analysis. The exact shape is illustrated in Fig. 1. The off-zones are chosen as fixed regions in galactic coordinates of identical shape and size as the on-zone. In the detector these shapes are observed with a time-shift of 1/4, 
$1 / 2$ and $3 / 4$ of a siderial day and therefore have the same visibility as the on-zone (see Fig. 2). Gaps in the data-taking and slight changes in the detector efficiencies can however lead to differences in the observed numbers of events in the on- and offzones. This effect was checked and found to be negligible. More specifically the numbers of events recorded in each of the off-zones were compared for various cut levels $\left(\Lambda^{\text {cut }}, E_{\text {reco }}^{\text {cut }}\right)$ and the differences were found to be within the statistical uncertainty. The approach of using on- and off-zones has also been used recently in a search for an enhanced neutrino emission from the southern sky [19]. The distributions of the parameters used for the cut optimisation, $\Lambda$ and $E_{\text {reco }}$, are shown in Fig. 3 for events coming from the off-zones with the preliminary event selection applied.

At energies of $100 \mathrm{TeV}$ and beyond the prompt neutrino flux from semi-leptonic decay of charmed particles might be a major contribution to the atmospheric neutrino background. This component is not present in the simulated data and the uncertainty on its flux is large. Due to the on- and off-zone approach this effect will however not alter the final result significantly.

\section{Cut optimisation}

To determine the optimal cut values for the dataset used in the update, the result of the 4year FB analysis needs to be taken into account. This first measurement has observed an average background of $n_{\mathrm{off}, 1}=11$ in the off-zones and $n_{\mathrm{on}, 1}=16$ events in the on-zone. The optimal cut values for the new data are obtained by minimising the average upper limit on the flux:

$$
\bar{\Phi}_{90 \%}=\Phi_{v_{\mu}+\bar{v}_{\mu}} \frac{\bar{s}_{90 \%}\left(b_{2} \mid n_{\mathrm{on}, 1}, n_{\mathrm{off}, 1}\right)}{s_{1}+s_{2}}
$$

where $s_{1}+s_{2}$ is the number of signal events simulated with the assumed neutrino flux $\Phi_{v_{\mu}+\bar{v}_{\mu}}$ from Eq. 2.5 in the whole data taking period used for the initial analysis $\left(s_{1}\right)$ and the update $\left(s_{2}\right)$. For a
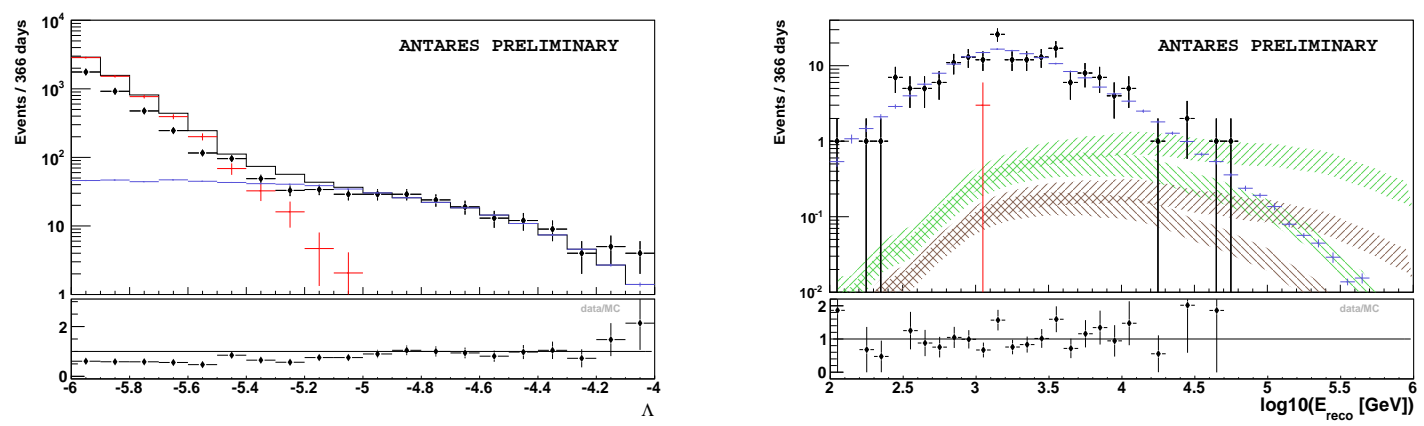

Figure 3: Off-zone distribution for measured (black points) and simulated events of the two reconstruction parameters used for optimisation of the signal sensitivity: On the left a transition of the main contribution from muons (red) to neutrinos (blue) is seen in the track fit quality parameter at $\Lambda \approx-5.2$. On the right the distribution of the reconstructed energy for $\Lambda>-5.1$ is compared to the distribution of simulated data. The signal flux (scaled up by a factor of 3 for easy comparison with the off-zones) for an $E^{-2}$ spectrum (green) and an $E^{-2.18}$ spectrum (brown) is also indicated for a $50 \mathrm{TeV}$ cut-off and no cut-off. The preliminary event selection mentioned in the text has been used. A scaling factor within the systematic uncertainties of the Bartol model has been applied to the atmospheric neutrino flux to allow for better agreement between measured data and simulation. 
Table 1: Resulting cut values $\left(\Lambda^{\text {cut }}, \log _{10}\left(E_{\text {reco }}^{\text {cut }}[\mathrm{GeV}]\right)\right)$ from the cut optimisation for an $E^{-2}\left(E^{-2.18}\right)$ neutrino spectrum on the left (right) with different cutoff energies. The average upper limits on the flux coefficient $\bar{A}_{90 \%}^{\alpha}$ are given in units of $1 \times 10^{-7} \mathrm{GeV}^{(\alpha-1)} \mathrm{cm}^{-2} \mathrm{~s}^{-1} \mathrm{sr}^{-1}$. In the last row, the cut values $\Lambda^{\text {cut }}=-5.14$ and $\log _{10}\left(E_{\mathrm{reco}}^{\text {cut }}[\mathrm{GeV}]\right)=4.03$ from the previous 4 -year analysis have been applied for all cut-off energies.

\begin{tabular}{l|rrrr|rrrr} 
& \multicolumn{4}{c}{$E^{-2}$ neutrino spectrum: } & \multicolumn{4}{c}{$E^{-2.18}$ neutrino spectrum: } \\
\hline$E_{v}^{\text {cutoff }}[\mathrm{TeV}]$ & $\infty$ & 500 & 100 & 50 & $\infty$ & 500 & 100 & 50 \\
\hline$\Lambda^{\text {cut }}$ & -5.34 & -5.16 & -5.16 & -5.34 & -5.16 & -5.16 & -5.16 & -5.32 \\
$\log _{10}\left(E_{\text {reco }}^{\text {cut }}[\mathrm{GeV}]\right)$ & 4.04 & 3.78 & 3.64 & 3.52 & 3.68 & 3.64 & 3.44 & 3.36 \\
\hline $\bar{A}_{90 \%}^{\alpha}$ & 3.73 & 5.60 & 9.41 & 13.9 & 29.3 & 38.3 & 59.0 & 78.3 \\
$\bar{A}_{90 \%}^{\alpha}($ cuts from $[10])$ & 3.78 & 5.74 & 10.0 & 15.5 & 30.0 & 40.2 & 65.3 & 91.3 \\
\hline \hline
\end{tabular}

known number of simulated background events in the new dataset, $b_{2}$, signal upper limits with a $90 \%$ confidence level, $\mu_{90 \%}$, are calculated following the approach in Ref. [21] to obtain the upper limit

$$
\bar{s}_{90 \%}\left(b_{2} \mid n_{\mathrm{on}, 1}, n_{\mathrm{off}, 1}\right)=\sum_{k=0}^{\infty} \mu_{90 \%}\left(k+n_{\mathrm{on}, 1}, b_{2}+n_{\mathrm{off}, 1}\right) \times \operatorname{Poisson}\left(k \mid b_{2}\right),
$$

which is an average over all possible numbers of events $k$ observed in the on-zone weighted by their Poisson probability. In the case of no discovery this best average upper limit represents the sensitivity of the ANTARES detector to the neutrino flux from the Fermi Bubbles [22].

The sets of cuts $\left(\Lambda^{\text {cut }}, E_{\text {reco }}^{\text {cut }}\right)$ optimising the average upper limit on the neutrino flux given in Eq. 4.1 and the respective flux normalisations are reported in Tab. 1 for an $E^{-2}$ neutrino spectrum. For an $E^{-2.18}$ spectrum the corresponding values are also given.

\section{Results}

The analysis used data taken in 2012 and 2013. In addition, two months of 2010 data which were not part of the 4-year analysis were added to the new analysis. Using only runs with low

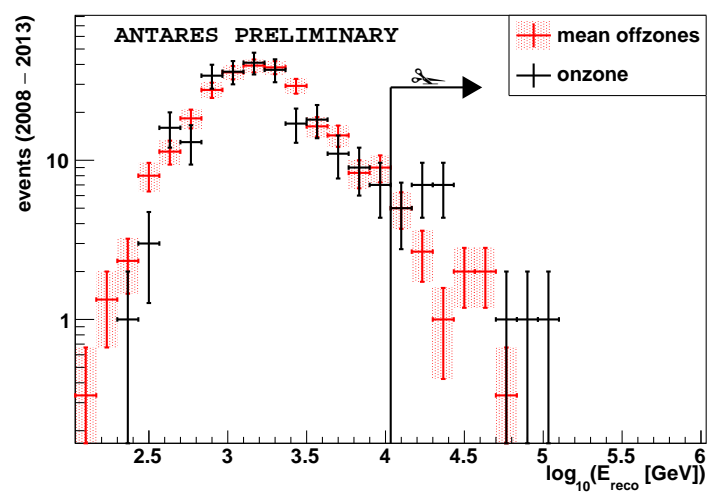

Figure 4: Distribution of the reconstructed energies for the six years of ANTARES data analysed with the preliminary event selection and the final cut on the reconstruction quality parameter, $\Lambda^{\text {cut }}>-5.14$, applied. From comparison with the off-zones an excess in the onzone can be seen at energies beyond the cut at $\log _{10}\left(E_{\text {reco }}[\mathrm{GeV}]\right)>4.03$. 

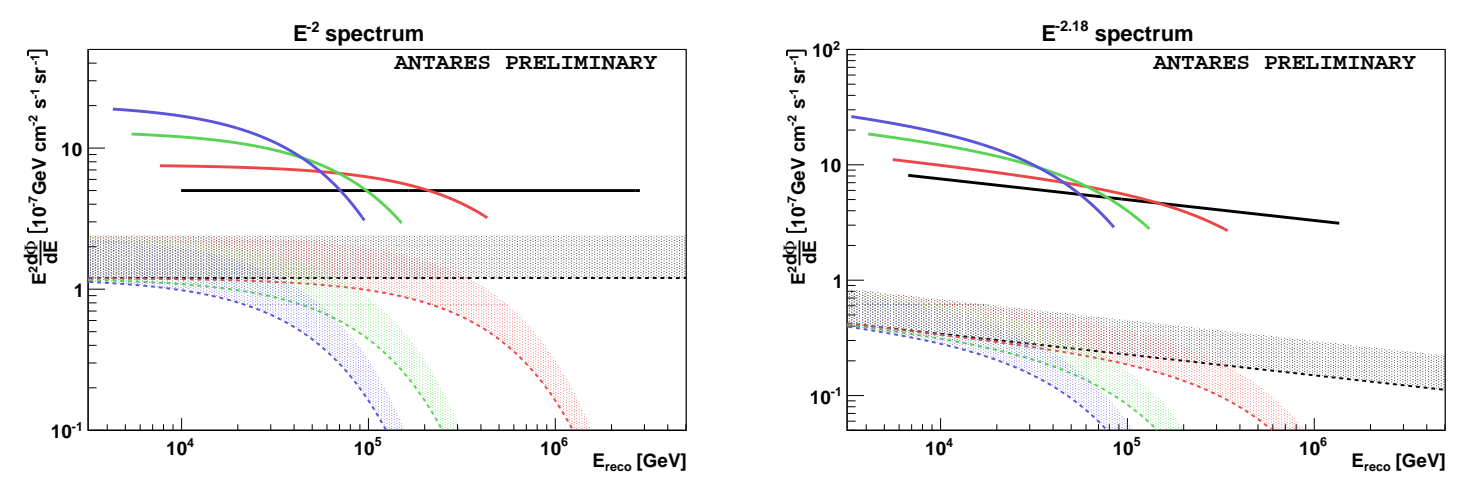

Figure 5: Upper limits on the neutrino flux from the Fermi Bubbles for different cutoffs (black: no cut-off, red: $500 \mathrm{TeV}$, green: $100 \mathrm{TeV}$, blue: $50 \mathrm{TeV}$ ) assuming a purely hadronic emission scenario. The shaded areas are the corresponding flux predictions. The limits are drawn for the energy range where $90 \%$ of the signal is expected.

optical background from bioluminescence and runs with good data taking conditions the total lifetime of the additional dataset sums to 366 days (c.f. 806 days in the 4-year analysis). Since the sensitivity does not change significantly when using the cuts of the 4-year analysis, i.e. $\Lambda>-5.14$ and $\log _{10}\left(E_{\text {reco }}[\mathrm{GeV}]\right)>4.03$, these cuts are also applied to the unblinded new dataset. In the three off-zones 1, 2 and 3 events are observed and add to the 33 background events in the 4-year analysis. In the region of the Fermi Bubbles 6 events are detected in addition to the 16 events in the first analysis. In Fig. 4 the energy distribution of the signal events in the on-zone is compared to the off-zones. Using the calculation from Ref. [23], the observed excess in the signal region is $1.9 \sigma$. The 90\% upper limits on the neutrino flux for the ANTARES data from 2008-2013 were calculated using the approach of Feldman\&Cousins [21] and are presented in Fig. 5. At the moment of writing this proceeding, a dedicated study of the systematic error is still ongoing.

\section{Conclusions and outlook}

In six years of ANTARES data the number of events observed in the Fermi Bubbles region shows yet no statistically significant excess over the background expectation. In the first 806 days, 16 events were found in the on-region with respect to 33 in the three off-regions, corresponding to an excess of $1.2 \sigma$. Adding the new data set of 366 days, the number of events in the signal region increases to 22 , and the background to $39 / 3$, with an excess of $\approx 1.9 \sigma$.

This analysis used track-like event signatures coming from charged current muon neutrino interactions. In contrast, charged current interactions with an electron in the final state and neutral current interactions produce showers of light with a much shorter extension in the forward direction. Recently developed methods provide an angular resolution of $5^{\circ}$ and below [24] with ANTARES. This makes extended sources like the Fermi Bubbles an ideal target for a combined analysis using track- and shower-like interaction channels. Also, in future, the KM3NeT detector as successor of ANTARES will improve the sensitivity to the neutrino flux from the FB by at least one order of magnitude [25]. 


\section{References}

[1] M. Su, T. Slatyer, and D. Finkbeiner ApJ 724 (2010) 1044, [arXiv: 1005.5480 ].

[2] S. L. Snowden et al. ApJ 485 (1997) 125.

[3] G. Dobler ApJ 750 (2012) 17, [arXiv:1109.4418].

[4] E. Carretti et al. Nature 493 (2013) 66, [arXiv: 1301 . 0512].

[5] R. M. Crocker and F. Aharonian Phys. Rev. Lett. 106 (2011) 101102, [arXiv:1008.2658].

[6] B. C. Lacki Monthly Notices of the RAS 444 (2014) L39, [arXiv: 1304.6137 ].

[7] S. Thoudam ApJ, Letters 778 (2013) L20, [arXiv: 1304 . 6972].

[8] P. Mertsch and S. Sarkar Phys. Rev. Lett. 107 (2011) 091101, [arXiv: 1104.3585$].$

[9] G. Dobler, I. Cholis, and N. Weiner ApJ 741 (2011) 25, [arXiv: 1102 . 5095].

[10] S. Adrian-Martinez et al. Eur. Phys. J. C74 (2014) 2701, [arXiv:1308. 5260].

[11] M. Ageron et al. Nuclear Instruments and Methods in Physics Research A 656 (2011) 11, [arXiv:1104.1607].

[12] C. James for the ANTARES Coll. Proceedings of the 34th ICRC ID 476 (2015).

[13] S. Adrian-Martinez et al. ApJ 760 (2012) 53, [arXiv: 1207.3105$].$

[14] M. Ackermann et al. ApJ 793 (2014) 64, [arXiv: 1407.7905 ].

[15] S. R. Kelner, F. A. Aharonian, and V. V. Bugayov Phys. Rev. D 74 (2006) 034018, [astro-ph/0606058].

[16] C. Lunardini, S. Razzaque, and L. Yang ArXiv e-prints (Apr., 2015) [arXiv:1504.0703].

[17] F. L. Villante and F. Vissani Phys. Rev. D 78 (2008) 103007, [arXiv: 0807 . 4151].

[18] J. Schnabel et al. Nucl. Instrum. Methods Phys. Res., Sect. A 725 (2013) 106.

[19] L. A. Fusco for the ANTARES Coll. Proceedings of the 34th ICRC ID 306 (2015).

[20] https://inspirehep.net/record/1250701/plots.

[21] G. J. Feldman and R. D. Cousins Phys. Rev. D 57 (1998) 3873, [physics / 9711021 ].

[22] G. C. Hill and K. Rawlins ApJ 19 (2003) 393, [astro-ph/0209350].

[23] T.-P. Li and Y.-Q. Ma ApJ 272 (1983) 317.

[24] T. Michael for the ANTARES Coll. Proceedings of the 34th ICRC ID 561 (2015).

[25] S. Adrian-Martinez et al. ApJ 42 (2013) 7. 